\title{
Bioenergía a partir de residuos ganaderos. Estado de situación en provincia de Buenos Aires
}

\author{
Bioenergy from livestock waste. Situation status in Province of Buenos Aires
}

\author{
Ada Graciela Nogar \\ Centro de Estudios Sociales de América Latina. Facultad de Ciencias Humanas, \\ Universidad Nacional del Centro de la provincia de Buenos Aires, Argentina \\ nogargraciela02@gmail.com \\ Carolina Chomicki \\ Centro de Estudios Sociales de América Latina. Facultad de Ciencias Humanas, \\ Universidad Nacional del Centro de la Provincia de Buenos, Argentina \\ carolinachomicki@gmail.com
}

José Luis Berdolini

Universidad del Noroeste de la Provincia de Buenos Aires, Argentina

jberdolini@gmail.com

\section{Resumen:}

El consumo de energía proveniente de fuentes fósiles se mantiene en crecimiento y sus consecuencias son inquietantes. Lo antes expresado se observa en el aumento de gases de efecto invernadero y en las dificultades de acceso/conectividad de poblaciones marginales y rurales. En este contexto, es necesario encontrar alternativas que atenúen el problema de la disponibilidad y colaboren con la diversificación de la matriz energética. La generación distribuida (GD) es un modelo que pondera las fuentes renovables, en el cual la producción de biogás a partir de la reutilización de residuos está ocupando y preocupando a actores públicos y privados. Este trabajo explora la producción de biogás en la Provincia de Buenos Aires a partir de un estudio de caso en Carlos Tejedor y reflexiona acerca del aprovechamiento de los residuos ganaderos, los procesos productivos/energéticos, la diversificación de la matriz energética y la reducción en la demanda de energías fósiles.

Palabras ClaVE: Energías renovables, Biogás, Oferta localizada, Ambiente, Buenos Aires.

\section{Abstract:}

Energy consumption from fossil sources grows and worries. This is based on environmental (greenhouse gases), social (access, inclusion) and territorial issues (shared ownership). In this context, there is a growing need to find alternatives that attenuate the problem of availability and collaborate with the diversification of the energy matrix. Distributed generation (DG) is a model that weighs renewable sources, in which the production of biogas from the re-use of waste is occupying and worrying public and private actors. This work explores biogas production in the Province of Buenos Aires and reflects on the use of livestock waste, production / energy processes, diversification of the energy matrix and reduction in the demand for fossil fuels.

KEYWORDS: Renewable energy, Biogás, Localized supply, Environment.

\section{INTRODUCCIÓN}

Desde principios del SXX las redes energéticas se constituyeron en el sistema neurálgico de las potencialidades de crecimiento territorial. Por un lado, su carácter transversal intersectorial endógeno a cada territorio y por otro, su importancia geopolitica según las fuentes y la disponibilidad de recursos (Nogar, A. G. 2017, p. 23). 
Además de revolucionar la historia industrial, el descubrimiento de los hidrocarburos colocó al petróleo, al carbón y al gas como los protagonistas del escenario energético mundial, lo que dio lugar a numerosos conflictos geopolíticos en torno al acceso, las reservas y los precios. De esta manera, la apropiación energética de los territorios se convierte en una dimensión sustancial para comprender los procesos de desigualdad socioterritorial, como también para explicar el crecimiento de algunos territorios. El informe del Club de Roma en 1972 puso en escena la situación crítica que vivirían los territorios si se mantenía la profundización del extractivismo, el crecimiento a costa de los recursos naturales y el aumento de la demanda de energías de origen fósil. En este contexto, aun los territorios con equilibrio en su matriz energética tienen el desafío de centrar los esfuerzos en acciones que prioricen los proyectos energéticos en consonancia con el modelo de transición energética impulsado desde la generación de energía descentralizada (De Gouvello, Durix, 2008, Moss et al., 2014; Alstone et al., 2015; Tirado Herrero et al., 2017), localizada y renovable. La generación distribuida (GD), entendida como "La producción de electricidad en o cerca del punto de uso, sin considerar el tamaño, la tecnología o el combustible utilizado, tanto fuera como dentro de una red" (WADE, 2017), es una estrategia que contribuye al desarrollo de los proyectos descentralizados.

Garantizar la accesibilidad, asequibilidad y seguridad energética en los territorios al margen de los tendidos, o donde el servicio es deficiente, o donde se aspira a sustituir fuentes fósiles; se convierten en objetivos a alcanzar, frente a la criticidad y limitaciones de los sistemas centralizados (Carrizo et al., 2017, p. 32).

Argentina no está exenta de esta problemática, a la cual se suma el aumento de la demanda energética, las insuficientes inversiones en infraestructura, los problemas de distribución y las políticas de los 90, que traspasaron al sector privado los servicios públicos. Todos ellos son factores que originaron el presente escenario de emergencia del sector eléctrico nacional (Decreto No134/2015). Las consecuencias se manifiestan en que haya población sin acceso a la red, y en el decrecimiento de la calidad sumado al incremento de los valores y a la reproducción de territorios depositarios de residuos, algunas veces tóxicos (Kozulj, 2003).

Cuando se indagan los antecedentes respecto al eje en análisis, se observa que la literatura que hace foco sobre las transformaciones en las redes energéticas desde las ciencias sociales es escasa, respecto de aquella que lo hace desde lo técnico (Dupuy, 1988; Curran, 2010). Por ello, se considera oportuno enriquecer los trabajos de investigación que hagan aportes desde análisis multidimensionales, transescalares e interdisciplinares, para profundizar los abordajes acerca de los impactos de la producción, la descentralización en la producción energética y la situación del consumo final de la energía (Mérenne-Schoumaker, 2007; Battiau, 2008). En este análisis será prioritario incluir los trabajos que analicen las jerarquizaciones y poderes de los actores insertos en el sistema energético que se encuentran en una relación de mutua dependencia (Bento et al., 2009). Los estudios relacionados con la diversificación/distribución de fuentes y producción (Tomei, Upham, 2009; De Nigris, Coviello, 2012; Molina, Rudnick, 2011, García Ochoa, 2014) aportarán herramientas para comprender la problemática, sumados a aquellos que hacen hincapié en el modelo de generación distribuida a partir de fuentes renovables (Ackermann, Andersson \& Söder, 2001; Gershenson y Kammen, 2015). Otros trabajos analizan las transformaciones territoriales producto de la diversificación de los procesos de generación (Furlán, 2010; AGN Auditoría General de la Nación, 2009; Pendón, Williams, Cibeira, Couselo, Crespi y Tittonel, 2017; Carrizo et al, 2017).

Los ejes enumerados no agotan el espectro de investigaciones realizadas, pero enuncian un horizonte respecto al tema en análisis, y hacen aportes al presente trabajo ${ }^{1}$ - que explora la producción de biogás a partir de residuos ganaderos mediante un estudio de caso en Carlos Tejedor Provincia de Buenos Aires- para analizar los obstáculos y alternativas de los procesos productivos/energéticos.

La recolección de datos primarios se realizó en 2017 a partir de encuestas a la población local y entrevistas semiestructuradas a informantes clave. La investigación se apoyó en la triangulación metodológica como campo propicio para la articulación de abordajes cuantitativos y cualitativos y el enfoque integral del objeto 
de estudio, privilegiando la perspectiva multidisciplinaria y multiescalar. Se identificaron las estrategias individuales y colectivas de los actores claves en el proceso, y en particular para este informe se sintetizan los obstáculos de la producción de biogás.

Los análisis realizados en este artículo se sustentan en la bibliografía trabajada y en el relevamiento de datos primarios y secundarios realizado.

\section{NUEVAS MIRADAS Y NUEVAS APROPIACIONES DE LOS ESPACIOS RURALES}

Discutir acerca de las funciones energéticas de los ER pampeanos en un contexto de expansión de la homogeneización productiva resulta como mínimo llamativo. Los actores construyen y/o participan de redes de diferente naturaleza y poder, al tiempo que despliegan estrategias a distintas escalas. Así, algunos alcanzan articulaciones con instancias de crecimiento a partir de nodos dinámicos con vinculaciones estratégicas, al momento que otros profundizan su dependencia reproduciendo su condición de marginalidad y deterioro en la que se materializan los conflictos sociedad/naturaleza. En este entramado, las cadenas de valor globalizadas intervienen en las neorrealidades rurales de acumulación territorial. Neorrealidades consideradas como un neologismo sostenido en los procesos de reconstrucción actual de los territorios, tal como lo expone Harvey (2004, p. 34).

El trastorno actual de la concepción espacio/tiempo es debido al proceso de innovación que provoca la aceleración de cambios, no sólo de productos, procesos, modos de trabajo y estilos de vida, sino también espaciales; una "destrucción creadora" que desintegra formas territoriales para crear otras nuevas, provocando una radical reorganización del espacio de relación.

Desde este abordaje teórico que visualiza nuevas territorialidades de los espacios rurales es posible enunciar que los mismos transitan por un entramado de integración funcional de actividades, actores, poderes y gobernanzas dispersas, dominados por redes difícilmente regulables que profundizan los procesos de fragmentación. Esta afirmación se sostiene en el creciente movimiento de reposición escénica de los recursos naturales y de la mano de obra, traccionados por el respaldo institucional (minería extractivista, revolución verde, profundización de los procesos hidrocarburíferos), el alza en los precios de los commodities, la disponibilidad de recursos escasos (agua, suelos, energía y minerales) y la concentración de dispositivos localizados de uso (infraestructuras extractivas, productivas, de distribución y de comercialización), que inducirían un crecimiento infinito, biofísicamente imposible.

Sin desconocer la complejidad del sistema, la intensificación de la degradación y la aceleración del agotamiento de los recursos, se considera en este artículo que es posible visualizar alternativas para atenuar la pobreza energética en América Latina (García Ochoa, 2014). El desarrollo de proyectos energéticos descentralizados favorece el forzoso tránsito hacia fuentes energéticas no fósiles, mientras que el interés de la comunidad internacional por analizar la escasez de recursos y su apropiación ilimitada, auspicia las gestiones que valorizan los recursos energéticos situados. Es aquí donde los espacios rurales se ven revisitados como territorios depositarios de fuentes energéticas descarbonizadas (Sanabria, 2018).

Como lo expresa Moreno Plata:

La adopción de estas fuentes energéticas implica la transformación de las relaciones campo-ciudad en virtud de que estos son dos piezas centrales y complementarias de este proceso: el territorio rural como el espacio idóneo y relevante para la generación energética, particularmente en el caso de la bioenergía, la energía solar o la eólica, que son las alternativas más prometedoras... (2011, p. 32).

En este entramado - producción primaria - residuos - reutilización - ambiente- son ejes que adquieren una significancia regional, mientras exigen un análisis de los conflictos y sinergias (tradeoffs analysis) entre beneficio económico y costo ecológico. Por ello, las políticas de ordenamiento y gestión territorial se tornan un instrumento idóneo para armonizar intereses socioeconómicos y ecológico-ambientales. De todas 
maneras, la convivencia de usos productivos con usos energéticos se manifiesta asociada a las características de los territorios. En algunos casos las tensiones se originan en la competencia por recursos naturales, como el suelo y el agua; en otros, se expresan acciones complementarias como en el caso de estudio enunciado.

\section{LAS BIOENERGÍAS EN EL CONTEXTO LATINOAMERICANO}

La documentación respecto a los antecedentes de la generación de biogás en América Latina y el Caribe es abundante, y muestra los diferentes contextos y estados de situación. La utilización de la tecnología de la biodigestión surge en los 70. Las pruebas piloto mostraron su funcionalidad técnica, pero fallaron en visibilizar la utilidad para el productor. En 2009 se organizó el Taller de Intercambio de Experiencias de Biodigestores en América Latina, y en el mismo año se formó la RedBioLAC (Red de Biodigestores para Latinoamérica y el Caribe). De modo simultáneo se formaron alianzas entre universidades, centros de investigación, empresas y ONGs para optimizar tecnologías y profundizar el desarrollo científico, técnico y legal.

En Perú el uso de la biomasa para generación de energía se aplica en:

I) Autoabastecimiento para la industria, II) introducción de la energía a la red eléctrica nacional a partir de los mecanismos de subastas de compra de energía proveniente de fuentes renovables iniciados el 2009, III) electrificación rural en lugares donde no hay acceso a la red nacional que representa aproximadamente a 5 millones de peruanos (FAO, 2013, p. 123).

En particular, Chile fomenta el biogás debido al aumento de pasivos ambientales, al cambio climático y al efecto invernadero (Ortiz et al., 2018).

En Uruguay, se diseñó el Plan Energético Nacional. Se definió el sistema energético bajo el rol directriz del Estado con el desarrollo de fuentes energéticas renovables.

En México, la acumulación, el manejo inadecuado y la incorrecta disposición final de desechos ganaderos ha provocado problemas ambientales relacionados con la contaminación del agua, suelo y aire (Weber, Rojas Oropeza, Torres Bernal, y Pampillón Gonzáles, 2012). Esto ha direccionado la creación de tecnologías para la biodigestión anaeróbica.

Por otro lado, Brasil es uno de los países pioneros en aplicar esta tecnología. Actualmente presenta proyectos de biogás distribuidos en diferentes lugares de su territorio (Milanez et al., 2018).

En Argentina el panorama es complejo e incierto. La matriz energética está ligada a los hidrocarburos. Según datos del CAMMESA (Compañía Administradora del Mercado Mayorista Eléctrico), su participación fue del $60 \%$ del consumo energético primario nacional para el año 2015, con destaque de gas y petróleo. Todo ello en un escenario de escasez y de transición hacia las energías descarbonizadas. Desde fines del siglo XX, pero sobre todo en las últimas décadas, se vienen desarrollando en los espacios rurales proyectos de generación de energías como biocombustibles a partir de cultivos de soja, maíz o caña de azúcar, el montaje de parques eólicos y plantas fotovoltaicas para la generación de electricidad, o el aprovechamiento de residuos agropecuarios en biodigestores para la generación de biogás.

En 2016 el PROBIOMASA (Proyecto para la promoción de la energía derivada de biomasa) junto a los integrantes de la Red de Biogás y los miembros del Programa de Energías Renovables del INTI presentaron el Relevamiento Nacional de Biodigestores. En base a esos datos algunos análisis realizados desde el INTI exponen que "el sector industrial entiende a la biodigestión anaeróbica como una etapa necesaria dentro del tratamiento de sus efluentes y no como una alternativa exclusiva de generación de energía" (2016, p. 23). Asume este informe que solo el $6 \%$ de las plantas relevadas fueron instaladas con fines energéticos, el resto prioriza la tecnología como solución para el tratamiento de efluentes. Respecto a esta situación, Tobares indica que la Argentina ha experimentado un avance en cuando a la producción de biodiesel y bioetanol, pero no en materia de biogás. La autora reconoce las potencialidades para la producción de biogás, la capacidad de descentralización a partir de materia orgánica y oportunidades de generación a diferentes escalas: 
La versatilidad de la digestión anaeróbica y el biogás hacen que también sea posible generar energía descentralizada y de diferente escala, como por ejemplo suministrar gas o luz eléctrica a hogares en el interior del país, utilizando pequeñas cantidades de residuos o efluentes (Tobares, 2012, p. 73).

Otros estudios observan que el biogás, en comparación con otras fuentes de energías renovables, genera energía durante las 24 horas del día mientras que "los molinos eólicos tienen una ocupación de 15 hasta 25 $\%$ del tiempo, los equipos solares, como los de fotovoltaica también trabajan con una ocupación limitante y solamente durante una parte del día" (Gruber, Hilbert, Sheimberg e INTA, 2010, p. 34).

En este contexto, el Ministerio de Energía y Minería ${ }^{2}$, a través de CAMESA, realizó en 2016 la convocatoria Abierta Nacional e Internacional "Programa RenovAr Ronda 1", para proyectos de generación de energía a partir de fuentes renovables según lo contenido en la Ley $\mathrm{N}^{\circ} 26.190$ y 27.191. Hasta mediados del 2018 , ejecutadas 3 rondas licitatorias, se adjudicaron 147 proyectos por $4.466,5 \mathrm{MW}^{3}$, correspondientes a 41 proyectos de energía solar, 34 de energía eólica, 18 de biomasa, 14 de aprovechamientos hidroeléctricos (pequeños), 36 de generación de biogás y 4 de biogás de relleno sanitario distribuidos en diferentes provincias (Ministerio de Energía y Minería, 2018).

De todas maneras, más allá de la presencia de recursos, Argentina se encuentra en una etapa de construcción de un marco regulatorio para orientar las exploraciones, las explotaciones y la descentralización de fuentes energéticas.

\section{BiodigeSTIÓN ANAERóBICA Y SU APROVECHAMIENTO ENERGÉTICO. ESTUDIO DE CASO.}

\section{Obstáculos y potencialidades}

Los desafíos de la generación descentralizada en general y de la producción de biogás en particular han sido enunciados en apartados anteriores. En el presente se expresan los obstáculos en la producción de biogás a partir del caso analizado en la unidad de producción ganadera La Micaela, ubicada a 2,5 km de la localidad de Carlos Tejedor, área con predominio de uso ganadero en sus diferentes formas, cría, recría, ciclo completo y engorde a corral, con manejo productivo intensivo y estabulado, que se diversifica para producir biogás a partir de la reutilización de residuos de sus 500 animales.

El desarrollo del proyecto bionergético originado en la articulación público/privada implicó tres años de negociaciones (2011-2014). La firma del convenio y la materialización de gestiones se instrumentaron con la participación del referente de la unidad productiva y la empresa Biogás, como privados, y PROINGED (Programa Provincial de Incentivos a la Generación de Energía Distribuida), la Cooperativa eléctrica de Carlos Tejedor y Municipio de Carlos Tejedor, como públicos. Para la compra del grupo electrógeno, la provincia de Buenos Aires otorgó un crédito a la cooperativa, que devuelve con el $20 \%$ de la bioenergía que se vende a la red. El restante ingreso por venta de energía le corresponde al productor.

A partir del análisis de los datos primarios relevados, se muestran en la figura $\mathrm{N}^{\circ} 1$ la distribución de las respuestas de las cinco (5) entrevistas realizadas a actores claves en la generación de biogás en Carlos Tejedor. Cada actor asignó determinada relevancia a los obstáculos mencionados, y de ellos, el número 5 fue el valor más relevante. 
FIGURA $N^{\circ} 1$

Principales obstáculos para la generación de biogás según actores claves

\begin{tabular}{|l|c|c|c|c|c|}
\hline & $\begin{array}{c}\text { Diversidad } \\
\text { de } \\
\text { intereses }\end{array}$ & $\begin{array}{c}\text { Falta de } \\
\text { legislación }\end{array}$ & $\begin{array}{c}\text { Complejidad } \\
\text { físico- } \\
\text { química del } \\
\text { biodigestor }\end{array}$ & $\begin{array}{c}\text { Inversión } \\
\text { inicial }\end{array}$ & $\begin{array}{c}\text { Adaptación e } \\
\text { innovación de } \\
\text { infraestructura }\end{array}$ \\
\hline Actor A & 5 & 4 & 2 & 1 & 3 \\
\hline Actor $B$ & 5 & 3 & 2 & 4 & 1 \\
\hline Actor $C$ & 5 & 4 & 2 & 3 & 1 \\
\hline Actor $\boldsymbol{D}$ & 3 & 5 & 4 & 1 & 2 \\
\hline Actor $E$ & 5 & 4 & 3 & 1 & 2 \\
\hline & 23 & 20 & 13 & 10 & 9 \\
\hline
\end{tabular}

Fuente: elaboración propia a partir de datos del trabajo de campo septiembre 2017

Estos obstáculos han condicionado el desarrollo del proyecto en Carlos Tejedor:

- Diversidad de intereses: la diversidad de intereses entre los actores es el obstáculo principal en el proceso. Es decir, la articulación entre los actores complejizó el proyecto; lo hizo lento y esto evitó la posibilidad de generar incentivos para reproducirse. La articulación entre actores públicos y privados implica desafíos y barreras constantes.

- Falta de legislación: otro obstáculo fue la falta de legislación, sumada a la falta de antecedentes en la temática, lo que fue determinante y provocó que cada parte del proceso fuera lento. Como menciona un actor clave: "Lo que pasó es que no había antecedentes legales respecto a este tipo de energía, no había experiencia entonces había que hacer una reglamentación y estipular tarifas, este fue el primer proyecto de biogás con este sistema” (Actor clave).

- Complejidad físico-química del biodigestor: dificultad asociada a parámetros de temperatura y de agitación dado el constante control que se requiere para mantener la comunidad bacteriológica en actividad para generar biogás.

- Inversión inicial y adaptación de infraestructura: la tecnología de la biodigestión requirió de una alta inversión inicial. En la unidad analizada se construyó el hormigón para recolectar de forma práctica los efluentes del feedlot, además del desarrollo de redes de conectividad, construcción del biodigestor y asistencia técnica computarizada, entre otras inversiones.

- Posibilidad de riesgo de la tecnología: aunque no fue mencionado en el caso de estudio de Carlos Tejedor, no deja de ser un obstáculo a la hora de producir energía a partir de residuos. Las paredes y el piso de hormigón presentan problemas estructurales, como agrietamientos, lo que requiere mantenimiento y control. Por otro lado, está forrado con lana de vidrio en el medio para que no pierda temperatura. Tiene un sistema de doble membrana que es la que siempre esta estirada para que el viento y lluvia no le hagan daño, y abajo hay otra que asciende y desciende con la presión del metano. Esto es estimulante a la generación de explosiones, en caso de no cumplir las normas 
de seguridad necesarias para trabajar con estos gases combustibles. Para evitar este riesgo deben realizarse las medidas preventivas, apropiadas para cada diseño particular de biodigestor, mediante accesorios de seguridad, instrucciones de operación y constante control durante el funcionamiento de esta tecnología. Según Bradfer, el mayor riesgo de la generación de biogás son sus características de inflamabilidad y explosividad al combinarse con el oxígeno contenido en el aire:

El biogás está compuesto de $\mathrm{CO} 2$ con efecto asfixiante (si $\mathrm{O} 2<18 \%$ ), y $\mathrm{H} 2 \mathrm{~S}$ tóxico (mortal si $>50 \mathrm{mg} / \mathrm{m} 3$ ). Estos dos gases son más pesados que el aire lo que implica un riesgo de acumulación en zonas bajas de recintos cerrados (cámaras, pozos, etc.). El peligro de asfixia o toxicidad del biogás puede resolverse mediante ventilación natural, detectores de gas y procedimientos para entrar en lugares de riesgo (detector portátil, equipo autónomo de respiración, etc.) (2002, p. 76).

En síntesis, estos proyectos tienen por delante desafíos de articulación regional entre la producción primaria, la producción industrial, la distribución (redes técnicas), la eficiencia, la comercialización y el consumo. El análisis realizado a partir del estudio de caso reafirma la hipótesis que los espacios rurales son revisados como territorios productores de energía y como enclaves de reutilización de residuos. Hacer visibles las transformaciones que se originan a partir de proyectos de generación energética desde fuentes renovables convierte en relevantes los supuestos que instalan el tema de las nuevas funciones de los espacios rurales, junto a otros que reclaman los límites al crecimiento indefinido.

La bibliografía consultada acerca de la generación eléctrica descentralizada expone que la misma a escalas diferentes fortalece las cadenas de valor, ya que incluye a productores/consumidores locales y regionales, ejerce un impacto en sus condiciones socioeconómicas y predica la utilización de un residuo contaminante. Nuestras investigaciones en desarrollo advierten que avizorar cambios profundos cortoplacistas de la matriz energética Argentina sería equivocado, ya que su aplicación implica consensos públicos/privados, embrionarios aún. En el caso particular de la producción de biogás a partir de residuos, los acuerdos se obstaculizan más cuando los actores/productores no acceden a créditos para realizar las inversiones, cuando su localización involucra otros desafíos y riesgos, cuando los marcos regulatorios, si existen, plantean parámetros poco accesibles.

Con esta investigación, se pretende poner a consideración de la comunidad académica, política y empresarial un tema que complejiza aún más los sistemas complejos. La producción de biogás se encuentra en su fase inicial, por lo que todos los aportes recorren la etapa de revisión, y el nuestro es uno más.

\section{REFERENCIAS}

Ackermann, T., Andersson, G., y Söder, L. (2001). Generación distribuida: una definición. Investigación de sistemas eléctricos, 57 (3), 195-204.

Argentina. AGN Auditoría General de la Nación. (2011). "Proyecto de energías renovables en mercados rurales dispersos (PERMER)”. Recuperado de https://www.agn.gov.ar/files/informes/2011_186info.pdf

Battiau, M. (2008). L'énergie: un enjeu pour les sociétés et les territoires. Ellipses.

Bento, N., Y Angelier, JP (2009). ¿La transición al hidrógeno está bloqueada por un bloqueo tecnológico a favor de los combustibles fósiles?

Bradfer, J. F. (2002). Riesgos y seguridad en el manejo del biogás en una planta de tratamiento de aguas servidas. In Congreso Interamericano de Ingeniería Sanitaria y Ambiental, 28 (pp. 1-8). FEMISCA.

Carrizo, S., Jacinto, G., Luciana, G., \& Nogar, G. (2017). Energías y territorios en Argentina: recursos no convencionales de principios de siglo XXI. Ciência \& Trópico, 41(1). 
Corrêa, R. L. (1994). Territorialidade e corporação: um exemplo. Território: globalização e fragmentação, 2, 251-256.

Cuaila, J. L. S., Coaquira, C. A., Perez, J. J. L., \& Cabanillas, C. R. Producción de biogás y biol a partir de excretas de ganado: experiencias en la ciudad de Tacna.

Curran, D. (2010). Systèmes énergétiques et espace géographique. Quelques remarques sur leurs interrelations. Historiens et Géographes, (409).

De Gouvello, C., Y Durix, L. (2008). Maximizar los usos productivos de la electricidad para aumentar el impacto de los programas de electrificación rural: una metodología operativa.

De Nigris, M., \& Coviello, M. (2012). Smart grids in Latin America and the Caribbean.

Dupuy, G. (1988) "Réseaux territoriaux. Transport \& communication".

Furlan, A. (2010). La reinvención de la geografía de la electricidad en el contexto de la transición energética contemporánea: Contribuciones a partir del caso de estudio de la costa atlántica bonaerense. In III Jornadas del Doctorado en Geografía 29 y 30 de septiembre de 2010 La Plata, Argentina. Desafíos teóricos y compromiso social en la Argentina de hoy. Universidad Nacional de La Plata. Facultad de Humanidades y Ciencias de la Educación. Doctorado en Geografía.

García Ochoa, R. (2014). Pobreza energética en América Latina.

Gruber, S., Hilbert, J., \& Sheimberg, S. (2010). Estudio de caso preliminar de generación eléctrica de 1 MWel con una planta de biogás de alta eficiencia. Buenos Aires: INTA N Doc BC-INF-16-10.

Harvey, D., Y Braun, B. (1996). Justicia, naturaleza y geografia de la diferencia (Vol. 468). Oxford: Blackwell.

INTA (2012). "Informe de la visita al establecimiento "La Micaela" Feedlot en Carlos Tejedor, Provincia de Buenos Aires". Recuperado de Programa Nacional de Agroindustria y Agregado de Valor de INTA: http://inta.gob.ar/sites/d efault/files/inta-establecimiento-micaela_feed_lot_biogas_2014.pdf

INTI (2017) "Relevamiento de Plantas de Biogás en Argentina". Recuperado de INTI: http://www.probiomasa.gob.a r/_pdf/Relevamiento.PROBIOMASA.pdf

Kozulj, R. (2005). La crisis energética de la Argentina: orígenes y perspectivas. Fundación Bariloche IDEE paper, 7.

Martí Herrero, J., Pino Donoso, M., Mendoza, G., Pedraza, G. X., Rodríguez Jiménez, L., \& Víquez Arias, J. (2016). Oportunidades para el desarrollo de un sector sostenible de biogestores de pequeña y mediana escala en LACRed de biogestores para Latinoamérica y el Caribe, REDBIOLAC (No. P06 17). RedBioLAC.

Mérenne-Schoumaker, B. (2011). Géographie de l'énergie. Acteurs. Belin.

Milanez, A. Y., Guimarães, D. D., Maia, G. B. D. S., Souza, J. A. P. D., \& Lemos, M. L. F. (2018). Biogás de resíduos agroindustriais: panorama e perspectivas.

Moreno Plata, M. (2011). Las funciones energéticas rurales para sustentar las zonas urbanas. Espacios Públicos, 14(32).

Nogar, A. G. (2017) “Accesibilidad e inclusión energética. Oportunidades y obstáculos desde las bioenergías” Dossier UNICen divulga. Recuperado de: Unicen.edu.ar.

Ortiz, D. L. P., Botero-Londoño, M. A., \& Botero-Londoño, J. M. (2019). Biomasa residual pecuaria: revisión sobre la digestión anaerobia como método de producción de energía y otros subproductos. Revista UIS Ingenierías, 18(1), 149-160.

Pendón, M. M., Williams, E. A., Cibeira, N., Couselo, R., Crespi, G., \& Tittonel, M. (2017, MAY). Energía renovable en Argentina: cambio de paradigma y oportunidades para su desarrollo. In IV Jornadas de Investigación, Transferencia y Extensión de la Facultad de Ingeniería (La Plata, 2017).

Sanabria, Á. (2018). La Economía del carbono: Una adicción de difícil tratamiento. Ciencia Política, 12(25), 51-65.

Tobares, L. (2013). La importancia y el futuro del biogás en la Argentina. Petrotecnia.

Tomei, J., Y UPHAM, P. (2009). Biodiesel argentino a base de soya: introducción a la producción e impactos. Politica energética, 37 (10), 3890-3898.

Weber, B., Rojas Oropeza, M., Torres Bernal, M., \& Pampillo Gonzalez, L. (2012). Producción de biogás en México. Red Mexicana de bioingeniería, AC, Mexico City Google Scholar. 


\section{PÁginAs WEB CONSULTADAS}

Biogás Argentina, 2017. “Energía sustentable, Biogás Argentina”. Lugar de publicación: Biogás Argentina: http://bio gas-argentina.com

Cámara de Diputados Provincia de Buenos Aires, 2015. Lugar de publicación: https://www.hcdiputados-ba.gov.ar/ proyectos/15-16d30870.pdf

CAMMESA (Compañía Administradora del Mercado Mayorista Eléctrico), 2017. Lugar de publicación: http://po rtalweb.cammesa.com

Diputados Argentina, 2017. Lugar de publicación: http://www.diputados.gov.ar/proyectos/buscador2016-99.html

Energía Estratégica, 2017. Lugar de publicación http://www.energiaestrategica.com

FAO (Food and Agriculture Organization), 2017. Lugar de publiación: http://www.fao.org

FAO (Food and Agriculture Organization),2013. Lugar de publicación: http://www.fao.org

INTI (Instituto Nacional de Tecnología Industrial), 2017. Lugar de publicación http://www.inti.gov.ar

MINISTERIO DE ENERGÍA Y MINERIA, 2017. Lugar de publicación: https://www.argentina.gob.ar/energiaym ineria

PROBIOMASA (2013). Lugar de publicación: http://www.probiomasa.gob.ar/sitio/es/

PROINGED (Programa Provincial de Incentivos a la Generación de Energía Distribuida), 2017. Lugar de publicación http://proinged.com.ar

WADE (World Alliance for Decentraliced Energy), 2017. Lugar de publicación: http://www.localpower.org

\section{Notas}

1 Este artículo se adscribe a proyectos desarrollados en el CESAL. "Integración productiva/energética de los territorios bonaerenses. Un desafío de hibridación entre usos productivos rurales y energías alternativas situadas" CICPBA y PICT 2017-2960 “Generación distribuida en Argentina. Energías para la inclusión y la transición”. (ANPCyT Investigadora Responsable Dra. Jacinto, G.; Investigadora Grupo responsable Nogar, A. G. 2018-2020).

2 Por ejemplo, los diputados Villalonga, Bardeggia y Tomassi, tras presentar sus propias iniciativas, estarían avanzando sobre un texto en común. Les corresponden respectivamente los proyectos de Ley (2965-D-2016), (5418D-2015) y (0097-D-2016). Fuente: http://www.energiaestrategica.com/ y http://www.diputados.gov.ar/proyectos/ buscador2016-99.html

3 Leyes $\mathrm{N}^{\circ} 26.190,27.191$ y el Decreto $N^{\circ} 31 / 2016$ 